\title{
Un Américain au Louvre ? L'architecte William Welles Bosworth et le réaménagement du musée du Louvre dans le cadre du « plan Verne » (1925-1939)
}

An American at the Louvre? The architect William Welles Bosworth and the reorganisation of the Musée du Louvre as part of the "plan Verne" (1925-1939)

\section{Églantine Pasquier}

\section{OpenEdition}

\section{Journals}

Édition électronique

URL : http://journals.openedition.org/cel/670

DOI : $10.4000 /$ cel.670

ISSN : 2262-208X

\section{Éditeur}

École du Louvre

\section{Référence électronique}

Eglantine Pasquier, « Un Américain au Louvre ? L'architecte William Welles Bosworth et le

réaménagement du musée du Louvre dans le cadre du « plan Verne » (1925-1939) », Les Cahiers de l'École du Louvre [En ligne], 11 | 2017, mis en ligne le 26 octobre 2017, consulté le 17 septembre 2019. URL : http://journals.openedition.org/cel/670 ; DOI : 10.4000/cel.670

Ce document a été généré automatiquement le 17 septembre 2019

\section{(c) (1) $\odot$}

Les Cahiers de l'École du Louvre sont mis à disposition selon les termes de la licence Creative Commons Attribution - Pas d'Utilisation Commerciale - Pas de Modification 4.0 International. 


\title{
Un Américain au Louvre? \\ L'architecte William Welles
}

Bosworth et le réaménagement $d u$ musée du Louvre dans le cadre du

« plan Verne » (1925-1939)

\author{
An American at the Louvre? The architect William Welles Bosworth and the \\ reorganisation of the Musée du Louvre as part of the "plan Verne" (1925-1939)
}

Églantine Pasquier

\section{NOTE DE L'ÉDITEUR}

Les traductions proposées ont été effectuées par l'auteur.

$\mathrm{Au}$ sortir de la Grande Guerre, le musée du Louvre se trouve dans une situation inconfortable. Les développements et remaniements successifs du musée, au sein d'un bâtiment qui n'a pas été conçu pour remplir cette fonction, ont entraîné un morcellement de la présentation des collections. Le département des Antiquités égyptiennes - mais ce n'est pas le seul dans ce cas - est par exemple installé sur deux étages et dans deux ailes différentes du Louvre, séparées l'une de l'autre de près de six cents mètres. Le musée est également trop à l'étroit dans ses locaux et il ne peut s'étendre car certaines ailes du palais sont occupées par d'autres institutions ${ }^{1}$. De ce fait, certaines collections ne peuvent être exposées, et celles qui le sont ne sont pas présentées de manière convenable. Le parcours et les œuvres sont donc difficilement lisibles pour les visiteurs, ce qui ne manque pas de faire l'objet de critiques dans la presse. Paul Lorquet écrit ainsi dans Le Quotidien en 1929 qu'un tour au Louvre, au département des Peintures, est à la fois « admirable et irritant ${ }^{2}$ ». Il reconnait la grande qualité des collections, mais reproche au Louvre un accrochage surchargé et un 
mauvais éclairage des œuvres. Il regrette également l'absence de courts textes introductifs donnant des clés de compréhension aux visiteurs, ainsi que l'absence de bancs dans les salles, ce qui rend la visite inconfortable. Cette situation dans laquelle se trouve le Louvre est d'autant plus fâcheuse qu'on assiste au même moment à la naissance de la "muséographie ", qui encourage la modernisation des musées et place l'éducation et le bien-être des visiteurs au cœur de ses préoccupations. Les conservateurs et historiens d'art français y prennent une part active et échangent à ce sujet avec des professionnels de musées de tous pays au sein d'institutions nouvellement créées comme l'Office international des musées (OIM), né en 1926. Chacun commence à mettre en œuvre, dans son institution, un accrochage allégé œuvres espacées, présentées sur une seule rangée - sur un fond neutre et clair. «Tout est ordonné, commode, mis en belle lumière ${ }^{3}$ ", précise Lorquet à propos des musées allemands et britanniques. En 1929, les premiers cours de muséographie font leur apparition à l'École du Louvre pour former les étudiants à ces nouvelles pratiques. Ce dynamisme de la recherche muséographique à laquelle la France prend part de manière active ne se reflète pourtant pas au Louvre, son musée le plus prestigieux et le plus renommé. Il devient donc urgent de remédier à ce problème.

\section{Henri Verne et la réorganisation du musée du Louvre}

\section{Qui est Henri Verne?}

2 C'est dans ce contexte de difficultés rencontrées au musée du Louvre mais aussi d'émulation muséographique à un niveau national et international qu'Henri Verne (1880-1949) est nommé directeur des Musées nationaux en octobre 1925. Il assure à ce titre la direction du musée et de l'École du Louvre, et occupe ce poste jusqu'en 1939, date à laquelle il est remplacé par Jacques Jaujard. Fort d'une double formation littéraire et juridique, Verne entre dans l'administration en 1904 en tant que rédacteur au ministère du Commerce. Deux ans plus tard, il devient chef de cabinet du ministre des Affaires étrangères Léon Bourgeois et suit ce dernier lorsqu'il devient ministre du Travail. En 1911, Verne publie un article sur l'organisation des loisirs ouvriers en Allemagne dans lequel il souligne le retard de la France à ce sujet et insiste sur l'importance de l'éducation ouvrière. La même année, il entre comme rédacteur aux Musées nationaux et l'année suivante en 1912, il devient sous-chef du secrétariat à la direction des Musées de France. En 1923, Verne publie deux volumes sur l'histoire du Louvre qui témoignent de son intérêt pour le musée et d'une bonne connaissance de son histoire. Cette publication n'est probablement pas étrangère à sa nomination en 1925 au poste de directeur des Musées nationaux. Verne est également, avec Henri Focillon (1881-1943), l'un des promoteurs de l'OIM, il est donc très au fait de toutes les avancées dans le domaine de la muséographie et ne peut que constater le retard du musée dont il a désormais la charge.

\section{L'action d'Henri Verne à la tête du Louvre}

3 Lors de son arrivée à la tête du Louvre, Verne fait preuve de dynamisme et d'une forte volonté de réforme. Pour répondre aux critiques qu'il estime justifiées, il souhaite moderniser le musée pour le rendre accessible à un public plus large. Il commence par faire éditer des «plans-guides» et "guides par l'image» du musée du Louvre, peu 
onéreux, destinés à faciliter la visite et à donner des clés de compréhension des œuvres. Mais il s'agit là d'une solution que l'on peut qualifier de palliative, qui vise à atténuer les désagréments liés à un parcours fragmenté et surchargé, et ainsi répondre aux problèmes rencontrés par les visiteurs à court terme. En parallèle, Verne s'attaque à la source du problème. Dès 1926, il commence à réfléchir avec Jaujard, alors secrétaire général des Musées nationaux et avec Camille Lefèvre, architecte en chef du Louvre, ainsi qu'avec les conservateurs de chaque département à un plan de regroupement et de redéploiement des collections au sein du palais. Les grandes lignes de ce projet sont présentées pour la première fois en 1927 dans le rapport annuel de Verne au ministre de l'Instruction publique ${ }^{4}$. Paul Léon, directeur général des Beaux-Arts, reconnaît que ce plan est tout à fait justifié, mais il ne dispose pas des crédits qui pourraient permettre son exécution. Les conservateurs doivent donc se contenter de petites améliorations au sein de chacun de leurs départements, en fonction des crédits disponibles. Verne et Lefèvre continuent toutefois à travailler sur un plan d'ensemble et en 1929, dans un rapport adressé au sous-secrétaire d'État aux Beaux-Arts, André François-Poncet, ils estiment que ce plan « est au point » et qu'il est "modestement encore, en voie d'exécution ${ }^{5}$ ». Ce plan, désormais connu sous le nom de "plan Verne », est rendu public en décembre 1929 par un article publié dans L'Illustration ${ }^{6}$ qui en explique les principales caractéristiques.

Le plan Verne se compose en réalité de deux projets (fig. 1). Un premier qui vise à regrouper les collections éparses de manière plus logique dans les espaces dont le Louvre dispose déjà au sein du palais. Un second qui vise lui aussi à regrouper les collections mais également à étendre la superficie accordée à chaque département dans l'hypothèse où le ministère des Finances quitterait les espaces qu'il occupe au sein du palais. L'aménagement muséographique prévu dans le cadre de ce plan témoigne de la volonté de mettre en œuvre les nouveaux principes muséographiques évoqués précédemment. Verne affirme en effet vouloir présenter les collections « en leur faisant bénéficier des exemples de réorganisation rationnelle et de développement que certains musées étrangers viennent de nous donner ». Les murs rouges, les riches décors en stucs, les dorures et l'accrochage à touche-touche sont totalement abandonnés. Cela suppose de faire une sélection, et de ne présenter que les œuvres les plus importantes de chaque genre, en reléguant les œuvres mineures dans des réserves qu'il souhaite toutefois ouvrir aux étudiants et aux chercheurs sur demande. Ces conceptions sont traduites dans les plans élaborés par Lefèvre, puis par Albert Ferran, qui lui succède à partir de $1930^{7}$. Verne exprime à plusieurs reprises dans ses publications vouloir, grâce à ce plan, rattraper le retard du Louvre par rapport à ses voisins étrangers. Il cite fréquemment en exemple le musée archéologique de Berlin qui consacre 120 millions de francs à sa réorganisation, le musée du Cinquantenaire de Bruxelles qui y accorde 70 millions de francs, ou encore le British Museum qui vient d'y consacrer 30 millions de francs. Il estime quant à lui qu'il faudrait 50 millions de francs pour mener à bien son plan de réorganisation du Louvre ${ }^{8}$. 
Fig. 1

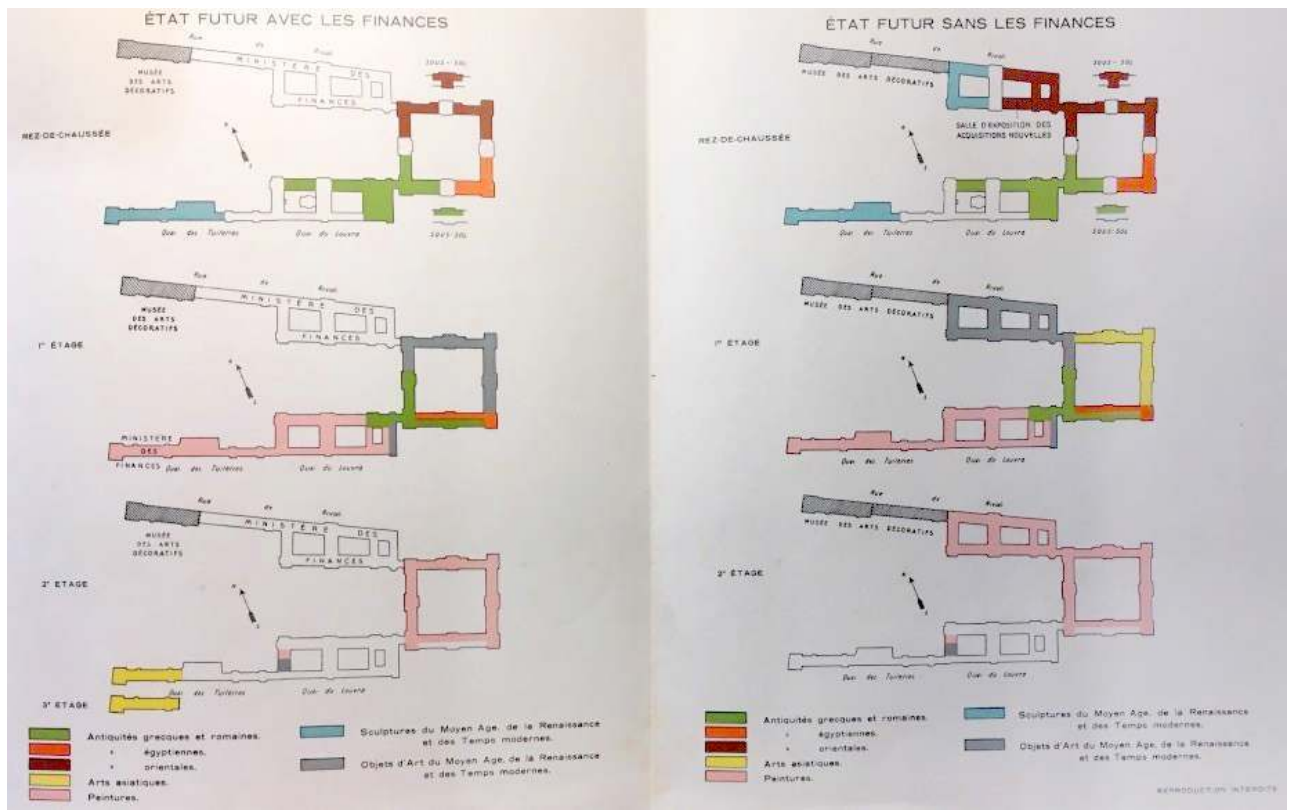

Plan d'extension et de regroupement des collections du musée du Louvre, Henri Verne, 1933, Pierrefitte-sur-Seine, AN, 20144794/35.

(C) Archives nationales, France

\section{Financer le «plan Verne »}

5 Au lendemain de la Grande Guerre, l'administration des Beaux-Arts a des moyens très limités et doit faire face au besoin de reconstruction de nombreux monuments historiques. Un rapport de Paul Léon, publié en 1922, estime que 785 édifices classés ont été endommagés par la guerre, dont 119 sont à reconstruire presque entièrement ${ }^{9}$. La loi sur les dommages de guerre du 17 avril 1919 prévoit la prise en charge des travaux de reconstruction par l'État, qui compte sur les réparations allemandes pour les financer. Toutefois, de nombreux monuments historiques qui n'ont pas été touchés directement par la guerre - c'est-à-dire bombardés - ont souffert indirectement des conditions de guerre : manque de chauffage, manque de matières premières et de maind'œuvre pour l'entretien courant. Ils se trouvent dans un état de délabrement parfois avancé et ne peuvent pas bénéficier de ces crédits de réparation, ce qui est par exemple le cas de Versailles. L'administration des Beaux-Arts doit donc aussi trouver une solution pour sauver ces monuments. Dans ces conditions, le réaménagement du Louvre n'apparaît pas comme une priorité car le palais n'a pas souffert de la guerre. Le clos et le couvert sont assurés, il peut donc abriter les collections sans les mettre en danger, même s'il est vrai que leur présentation pourrait être améliorée. L'appel à des fonds privés semble donc être la seule solution pour réunir rapidement les 50 millions de francs nécessaires au financement du plan de réaménagement. Léon et Verne pensent en particulier à des fonds américains, ces derniers s'étant montrés particulièrement généreux envers le Louvre et le patrimoine français de manière générale. Par exemple, quelques mois auparavant, c'est une donation de Walter Guy Mortland qui a permis de réaménager une nouvelle salle de l'École du Louvre, dans un sous-sol du quai des Tuileries, ainsi que le musée épigraphique gréco-romain. Léon décide alors de s'adresser à un Américain qu'il connaît bien, William Welles Bosworth 
(1869-1966), architecte installé en France depuis quelques années, pour l'aider dans sa recherche de mécène.

\section{La recherche d'un financement américain}

\section{Qui est William Welles Bosworth?}

6 Bosworth s'est formé au métier d'architecte au Massachusetts Institute of Technology (MIT) à Boston et à l'École des beaux-arts de Paris, où il est admis en 1897. Il retourne ensuite aux États-Unis pour ouvrir sa propre agence et travaille à de nombreuses reprises pour la famille Rockefeller, ce qui lui permet de tisser des liens d'amitié avec le philanthrope John D. Rockefeller Jr. (ci-après Junior), fils du fondateur de la Standard Oil, qu'il conseille dans le domaine artistique et architectural. En 1924, lorsque Junior fait don de la somme d'un million de dollars à la France pour restaurer les palais de Versailles et Fontainebleau et la cathédrale de Reims, il demande à Bosworth de s'installer à Paris pour superviser les travaux et le représenter auprès de l'administration. C'est dans ce cadre qu'il est amené à collaborer étroitement avec Paul Léon. Les travaux de restauration s'achèvent en 1936, mais Bosworth décide de rester en France, où il vivra jusqu'à la fin de sa vie. Au cours de cette période, il construit peu, mais reste très actif dans les cercles politiques et artistiques parisiens qu'il connaît bien. Il devient également l'une des figures majeures de la communauté américaine de Paris qu'il contribue à animer et dans laquelle il est très bien introduit.

\section{Pourquoi faire appel à William Welles Bosworth ?}

7 Le moment où Henri Verne et Paul Léon recherchent un financement pour le Louvre coïncide avec un élan de générosité sans précédent de Junior envers le patrimoine français. En mai 1927, le milliardaire effectue une seconde donation en faveur de Versailles, Reims et Fontainebleau, cette fois-ci d'un montant de deux millions de dollars (soit environ 40 millions de francs). En mai 1928, il annonce qu'il financera la restitution des vitraux du chœur de la cathédrale de Chartres, et quelques mois plus tard, en juillet 1928, il effectue une donation de deux millions de dollars pour financer la construction de la Maison internationale de la Cité universitaire de Paris. La fréquence et l'importance des libéralités de Rockefeller ont très probablement poussé nos deux hommes à penser qu'il pourrait aussi être intéressé par le financement du plan Verne. Pour chacun de ces dons, c'est Bosworth - déjà familier des rouages de l'administration française - qui est impliqué dans les négociations préparatoires et désigné comme représentant plus ou moins officiel de Junior. Aussi, fin 1928, Léon décide de s'adresser directement à lui pour qu'il parle du projet du Louvre au philanthrope. Bosworth prend immédiatement contact avec Verne pour se familiariser avec son plan, ce dont atteste l'extrait de cette lettre que Verne adresse à Lefèvre :

M. Paul Léon a déjà entretenu de notre programme de travaux à faire au Louvre votre collègue, l'architecte de M. Rockefeller, M. Bosworth. Celui-ci considère comme très vraisemblable l'acceptation de M. Rockefeller de subventionner un projet concernant le Louvre. Il s'est offert à venir dès qu'on le voudrait, le plus tôt possible, examiner le palais et sans doute aussi étudier nos desseins et vos dessins ${ }^{10}$. 
Cette visite de Bosworth au Louvre s'est déroulée le 28 décembre $1928^{11}$. Fin 1928 donc, Bosworth est familier du projet, il a rencontré ses principaux acteurs, et attend désormais le moment propice pour le présenter à Rockefeller.

\section{John D. Rockefeller Jr. étudie le projet}

8 Ce moment arrive le 19 mars 1929, lorsque Junior est brièvement de passage à Paris sur le trajet du retour d'un voyage en Égypte. Il demande à Bosworth de le conduire à Versailles et à Chartres pour inspecter les travaux qu'il finance. C'est à ce moment, alors qu'ils sont dans la voiture, que Bosworth lui présente le plan Verne ${ }^{12}$. Rockefeller est intéressé, mais il a pour habitude de ne jamais prendre de décision sans avoir sollicité au préalable l'avis de plusieurs conseillers philanthropiques. Il demande donc à Bosworth de contacter Abraham Flexner et Arthur Woods pour qu'ils donnent leur avis sur le projet. Abraham Flexner (1866-1959) a longtemps travaillé pour la famille Rockefeller au sein du General Education Board, une branche de leur empire philanthropique consacrée au développement de l'éducation. Il est à Paris à la fin de l'année 1928 et avait accompagné Bosworth lors de sa visite au Louvre le 28 décembre. Sollicité pour donner son avis le 20 mars 1929, il envoie son rapport à Rockefeller dès le 27 mars et émet un avis favorable au financement du projet en raison de l'intérêt éducatif que présente pour lui le Louvre.

Le Louvre n'appartient pas seulement à la France, mais au monde. Chaque peintre américain, chaque individu qui souhaite développer son sens esthétique, doit connaître et étudier ses riches trésors. [...] Néanmoins, aussi riche qu'il soit, ses collections ne peuvent pas être convenablement et intelligemment présentées et utilisées sans une réorganisation très considérable. [...] Il est évident que [...] l'utilité de leur magnifique collection [est] entravée par des conditions auxquelles seul l'argent peut remédier. Par conséquent, un don au Louvre, de mon point de vue, serait une manière très efficace d'aider au développement du goût et des compétences américaines, et bien sûr, il bénéficierait aux Français et également aux autres nations ${ }^{13}$.

La deuxième personne à qui Rockefeller s'adresse est Arthur Woods (1870-1942). Trustee de la Rockefeller Foundation et du General Education Board, il a déjà conseillé le philanthrope à de nombreuses reprises, notamment sur le vaste projet de restauration de Colonial Williamsburg en Virginie qu'il a entrepris en 1927. Il émet quant à lui un avis défavorable au financement du projet. Il reconnaît que la demande est justifiée car le Louvre a effectivement besoin d'une réorganisation, mais il pense toutefois «qu'il s'agit d'un projet gouvernemental qui est en cours de réalisation, qui sera lentement accompli dans le cours naturel des évènements, et qui ferait mieux d'être laissé au gouvernement pour qu'il le réalise à son rythme ». Il ajoute « je penserais différemment à ce sujet si les œuvres d'art souffraient de détérioration, comme ce fut le cas à Versailles, par exemple, ou si le bâtiment qui les abritait avait un toit qui fuit ou était dans un état tel qu'il entraînerait la détérioration ou des risques pour les œuvres qu'il abrite $\aleph^{14}$. Il est important de préciser ici que si les conseillers auxquels Junior fait appel sont affiliés d'une manière ou d'une autre à des organisations philanthropiques créées ou gérées par la famille Rockefeller, les donations faites à Versailles, Reims, Fontainebleau, Chartres et celle qui est envisagée pour le Louvre sont effectuées sur ses fonds personnels. Elles témoignent donc d'un intérêt particulier pour les questions culturelles et patrimoniales. Le philanthrope décide toutefois de ne pas financer le plan 
Verne, se rangeant ainsi derrière l'avis de Woods. Il informe Bosworth de sa décision dans une lettre datée du 28 mai 1929:

J'ai examiné attentivement et avec soin le projet que vous m'avez soumis pour la réorganisation et une meilleure utilité du musée du Louvre. Il n'y a aucun doute sur le besoin ou sur la valeur qui serait de cette manière ajoutée au musée et à son utilité générale. Ce projet doit être réalisé, et, comme vous le dites, est en train d'être réalisé petit à petit par le gouvernement. J'aurais souhaité pouvoir contribuer à l'accélération de leur exécution, mais pense que ce n'est pas la meilleure des choses d'entreprendre de nouveaux projets en France, au moins pour le moment. De plus, si j'étais disposé à faire une nouvelle contribution, je serais plus enclin à compléter les sommes déjà dévolues à la préservation et la restauration de Versailles ${ }^{15}$.

Cette décision est finalement conforme à la conception que Junior se fait de la philanthropie et sur laquelle il insiste à chaque nouvelle donation. Il ne souhaite en aucun cas remplacer l'État ou l'organisme auquel il vient en aide, mais souhaite les soulager temporairement lorsqu'ils se trouvent dans une situation difficile, le temps qu'ils puissent se remettre de leurs difficultés et reprendre le flambeau. C'est pour cette raison qu'il étudie aussi précisément la situation à chaque fois qu'une demande lui est faite. Il estime donc, pour le Louvre, que ça n'aurait pas été rendre service au gouvernement que de le remplacer dans une tâche qu'il avait déjà commencé à entreprendre.

\section{La recherche infructueuse d'un autre mécène américain}

10 Malgré le refus de Rockefeller, ni Paul Léon ni Henri Verne n'ont perdu l'espoir de trouver un financement américain pour le Louvre et Bosworth poursuit ses recherches. Quelques mois plus tard, il contacte à nouveau Henri Verne, « Toujours à la recherche d'un ami américain pour le Louvre, j'ai trouvé une piste qui pourrait donner d'excellents résultats, et j'aimerais beaucoup vous amener la personne intermédiaire pour examiner la situation rapidement, mercredi après-midi à quatre heures. Pouvezvous vous rendre libre à ce moment-là, car c'est la seule heure dont ce Monsieur puisse disposer avant son départ pour New York ${ }^{16}$ ? " Ce «Monsieur ", dont l'identité reste inconnue, a lui aussi décidé de ne pas financer le projet. Mais cela montre bien d'une part, que Bosworth est toujours impliqué dans la recherche d'un financement pour le plan Verne, même après le retrait de Rockefeller. Et d'autre part, cela confirme qu'il a joué un rôle d'intermédiaire pour la philanthropie américaine en France. Du moins, il était perçu comme tel par l'administration des Beaux-Arts qui l'a sollicité à d'autres reprises dans les années qui ont suivi. Finalement, la recherche d'un mécène Américain s'étant avérée infructueuse, Henri Verne a réussi à convaincre les représentants successifs des Beaux-arts et certains députés de faire voter par le Parlement l'attribution de tranches du plan d'outillage national au musée du Louvre à partir de 1931. Ce sont donc les crédits de l'État qui ont permis de mettre en œuvre la réorganisation du musée. Et si l'on en croit Bosworth, le fait que tous les plans aient été " approuvés par l'architecte de Rockefeller » aurait favorisé le vote de ces crédits en faveur du Louvre ${ }^{17}$. 


\section{Quelques pistes sur une possible implication de William Welles Bosworth}

\section{Bosworth, un conseiller non officiel ?}

11 Les tentatives de Bosworth pour aider le Louvre à trouver un mécène américain n'ont pas abouti, mais il est probable qu'il soit resté associé de manière informelle à la mise en œuvre du plan Verne. Plusieurs éléments vont en ce sens. En 1930, il reçoit une invitation pour l'inauguration de la nouvelle salle de cours de l'École du Louvre financée par Mortland ${ }^{18}$ et en 1934, il est présent à l'inauguration de la cour du Sphinx, nouvellement aménagée dans le cadre du plan Verne (fig. 2). Si ces deux éléments ne prouvent pas en soi l'implication certaine de Bosworth dans la mise en œuvre du plan de réaménagement, ils témoignent du fait qu'il gravitait autour du projet et de ses acteurs. Il n'est donc pas impossible d'imaginer qu'il ait pu être sollicité, en tant que conseiller, pour donner son avis sur certains éléments architecturaux ou muséographiques en raison de sa proximité avec les principaux acteurs du projet et de sa bonne connaissance des musées américains. Toutefois, s'il y a eu activité de conseil, elle s'est déroulée de manière informelle car son nom ne figure sur aucun plan, aucun document ou aucune correspondance en relation avec la mise en œuvre du plan Verne émanant des archives de l'agence d'architecture du Louvre ou de celles du musée après la période de recherche de financement. Une raison qui peut expliquer ce caractère non officiel est que le nom de Bosworth est tellement associé à celui de Rockefeller, que tout rôle officiel aurait pu laisser penser à une implication du philanthrope dans le réaménagement alors que ce n'était pas le cas. 
Fig. 2

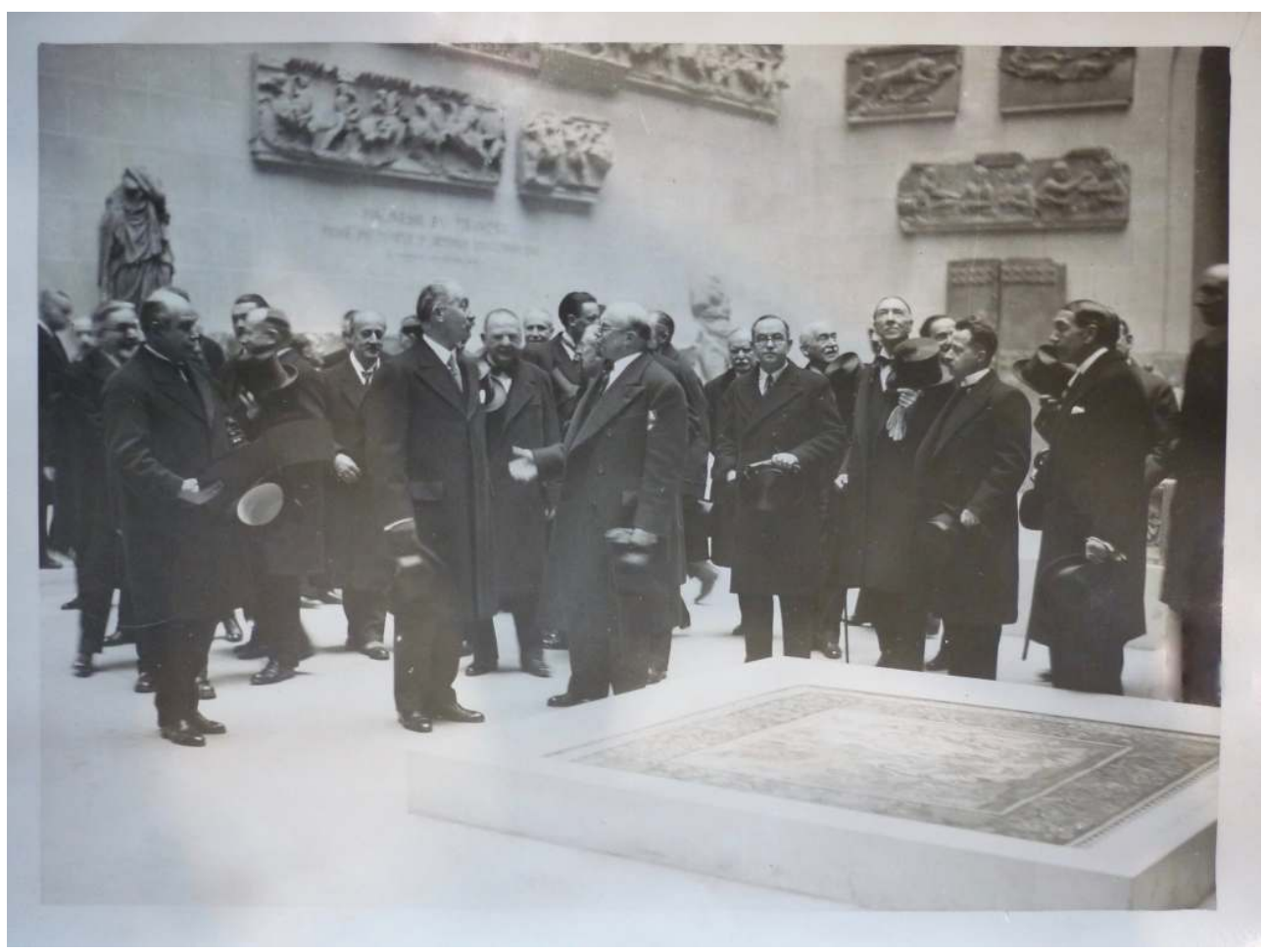

Inauguration de la nouvelle salle Magnésie et Millet dans la cour du Sphinx, photographe anonyme, 1934, Pierrefitte-sur-Seine, AN, 20144794/37. Bosworth est la troisième personne en partant de la droite (il regarde en l'air)

(c) Archives nationales, France

\section{Une certaine proximité avec les acteurs du projet}

Revenons maintenant sur la proximité de Bosworth avec les principales personnalités impliquées dans le plan Verne. Nous l'avons vu, du fait de son statut de représentant de Rockefeller dans divers projets de restauration en France, Bosworth rencontre fréquemment Paul Léon. Il rencontre aussi de manière régulière Pierre Paquet, inspecteur des Monuments historiques dans le cadre du travail de restitution des vitraux du chœur de la cathédrale de Chartres. Or, Paquet est également membre d'une sous-commission des Monuments historiques consultative, créée pour donner son avis sur les travaux entrepris dans le cadre du plan Verne, les deux hommes ont donc pu évoquer les travaux du Louvre ensemble. Bosworth était également proche d'un certain nombre de conservateurs du Louvre. Dans l'une des lettres qu'il écrit à Junior en 1930, il l'informe qu'il a récemment assisté à un dîner au cours duquel il était " assis à côté du conservateur du Louvre ${ }^{19}$ » (sans préciser lequel), et que ce dernier l'avait informé de la mise en vente d'une célèbre collection qui pourrait intéresser le philanthrope. Il est probable qu'au cours d'un tel dîner, les travaux en lien avec le plan Verne aient également été abordés. Bosworth invite aussi régulièrement des personnalités du monde des musées et des beaux-arts pour qu'ils donnent une conférence dans le cadre des dîners ou déjeuners qu'il organise au University Club, club américain de Paris dont il est le fondateur et le président. Le 28 avril 1936, c'est Paul Jamot, conservateur au département des Peintures du Louvre, qui est invité à donner une conférence, en présence de Georges Huisman, directeur général des Beaux-Arts. En décembre 1937, 
Jean Zay, ministre de l'Éducation nationale et des Beaux-Arts du Front Populaire est invité d'honneur d'une soirée, et le 9 novembre 1938 c'est Paul Léon qui est invité à son tour $^{20}$. Le contenu de ces conférences et la teneur des discussions qui ont suivi ne sont pas connus, mais il est possible que les travaux en cours au Louvre aient été évoqués. Un dernier élément qui viendrait soutenir cette hypothèse d'une activité de conseil informelle de la part de Bosworth est son lien supposé avec Albert Ferran, architecte du Louvre à partir de 1930. Il est possible que les deux hommes se connaissent déjà depuis plusieurs années au moment où le plan Verne commence à être mis en œuvre. Ferran est né aux États-Unis de parents français et après sa formation à l'École des beaux-arts de Paris, il a enseigné au département d'architecture du MIT pendant deux ans, de 1922 à 1924. Le MIT est l'école dans laquelle Bosworth s'est formé, mais il a surtout été l'architecte de son nouveau campus, installé sur les bords de la Charles River à Cambridge, face à Boston. Le bâtiment principal est inauguré en 1916, mais jusque dans les années 1920 et même au-delà, le campus est encore un immense chantier et Bosworth réalise de nombreuses additions, il est donc encore actif au MIT entre 1922 et 1924 lorsque Ferran s'y trouve. Il serait surprenant que Bosworth, architecte des nouveaux bâtiments, francophone et francophile, et Ferran, professeur d'architecture, lui-même français et ancien élève comme Bosworth de l'École des beaux-arts, ne se soient pas rencontrés. Cette proximité supposée des deux hommes aurait dans tous les cas favorisé leur échange à propos du réaménagement du Louvre dans le cadre du plan Verne.

\section{Une bonne connaissance du Louvre et des musées américains}

Bosworth disposait également d'une bonne connaissance du Louvre et des musées américains de construction récente, ce qui pourrait aussi expliquer pourquoi ses conseils auraient été sollicités. En tant qu'ancien élève de l'École des beaux-arts, il a probablement fréquenté assidûment le musée du Louvre, d'autant plus qu'il fut élève dans l'atelier de Gaston Redon, frère du peintre Odilon Redon, qui à ce moment-là était architecte du palais. Au moment où Bosworth étudiait dans son atelier, en 1900, Redon travaillait à l'aménagement de l'ancienne salle des Sessions pour y présenter la série des tableaux de Rubens illustrant la vie de Marie de Médicis. Quelques années plus tard, en 1905, c'est également Redon qui a installé le musée des Arts décoratifs dans le pavillon de Marsan. Mais c'est surtout la bonne connaissance qu'avait Bosworth des musées américains qui a pu être mise à profit dans le cadre du plan Verne. Pour mieux comprendre cette familiarité avec les musées américains, il faut encore une fois revenir à Rockefeller. En 1925, Junior décide de financer un projet de musée des Antiquités égyptiennes au Caire pour remplacer le bâtiment construit par Marcel Dourgnon place Tahrir et inauguré en 1902. Il demande à Bosworth d'en être l'architecte. Ce projet comprenant un musée, un centre de recherche (fig. 3) et un fonds de dotation permettant d'assurer son fonctionnement fut présenté «clé en main » par Rockefeller au roi Fouad, comme un cadeau fait au peuple égyptien. Il fut refusé car le roi craignait - à juste titre - la mainmise des Américains sur le patrimoine égyptien ${ }^{21}$, ce projet ne vit donc jamais le jour. 
Fig. 3

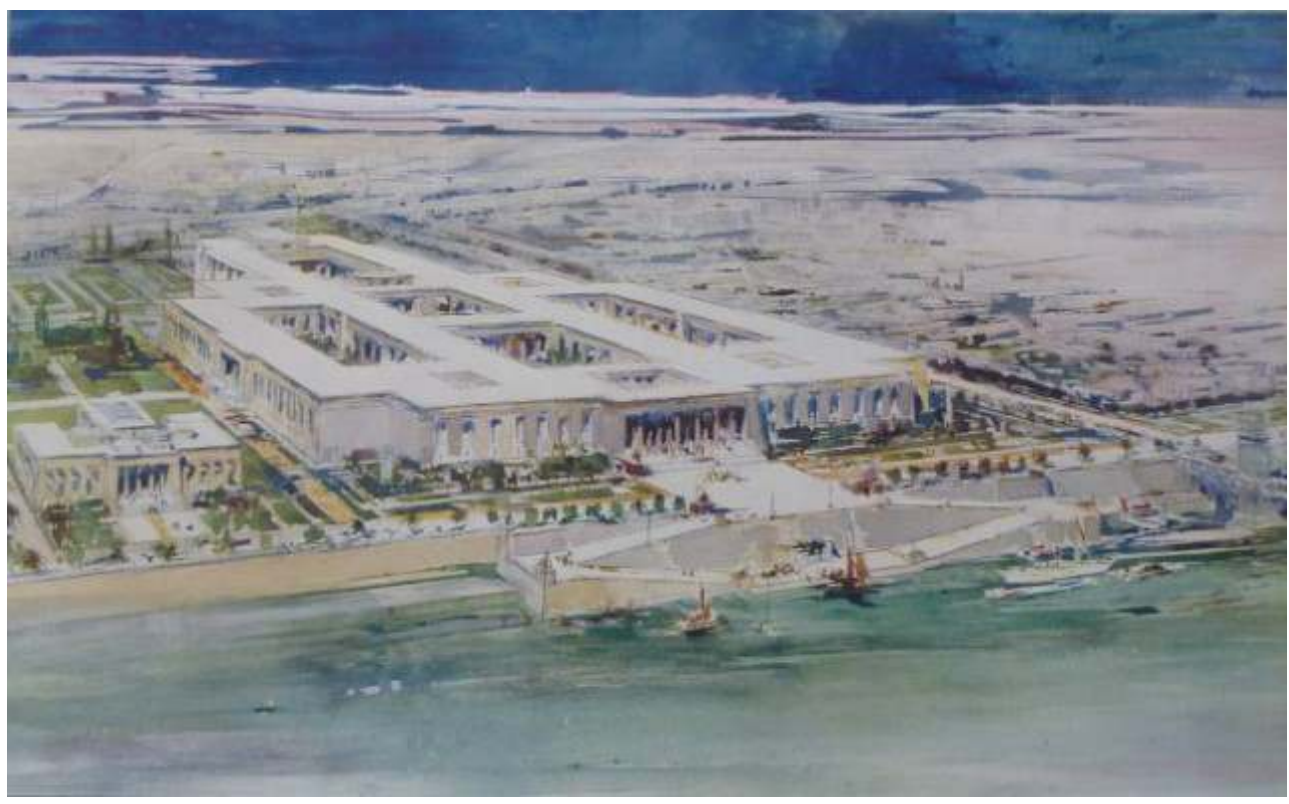

Projet de musée (à droite) et de centre de recherche (à gauche) au Caire, aquarelle de William Walcot d'après un dessin de Bosworth, 1925, dans The New Egyptian Museum and Research Institute at Cairo, planche 1, RAC, Folder 258A, Box 25, Series E, OMR - Office of the Messrs. Rockefeller

Toutefois, dans le cadre de sa préparation, Rockefeller a financé un voyage d'étude des principaux musées américains du Nord-Est à Bosworth et aux autres membres du comité d'organisation de ce musée, l'objectif étant d'étudier les questions d'éclairage et d'aménagement intérieur car «les nouveaux musées américains ont maintenant rassemblé une expérience significative, bien plus que celle qui est à présent disponible en Europe, ou qui serait connue, par exemple, de tout architecte anglais ou européen ${ }^{22}$ ». C'est ainsi qu'en septembre 1925, Bosworth visite le Metropolitan Museum of Art de New York, le Museum of Fine Arts de Boston, le Cleveland Museum of Art, le Minneapolis Institute of Art et l'Art Institute de Chicago. Il étudie également les plans de Paul Cret pour le Detroit Institute of Arts dont la construction vient de démarrer. Bosworth n'a pas laissé d'écrits à propos de cette tournée, il existe en revanche un livret illustré et commenté décrivant le musée projeté au Caire ${ }^{23}$. Il est intéressant de comparer le projet avec les modifications effectuées au Louvre dans le cadre du plan Verne quelques années plus tard, car certaines similitudes peuvent être constatées. Dès les premières lignes de la présentation, les choses sont clairement exprimées, le musée du Caire - tout comme le Louvre quelques années après - se veut être à la pointe de la modernité : « Le nouveau bâtiment [...] sera profondément moderne dans son éclairage, son organisation, la distribution de son espace et ses méthodes de construction ${ }^{24}$. » La suite de la description nous apprend qu'une présentation chronologique des collections, censée communiquer une idée de progrès de l'art est envisagée, mais que seulement les meilleures œuvres seront présentées pour éviter "la congestion » de l'ancien musée. Ce nombre réduit d'œuvres ainsi que leur installation dans de nouvelles salles spacieuses et lumineuses permettra ainsi "à chaque ancien chef-d'œuvre d'exister pour lui-même et, non entravé par des conditions d'encombrement, de dévoiler sa valeur individuelle en tant que chef-d'œuvre ${ }^{25}$ " (fig. 4). Il s'agit là de la même idée que celle qui a présidé au nouvel aménagement du Louvre, un accrochage 
allégé, sur fond clair, permet de mieux mettre en valeur une sélection d'œuvres (fig. 5). Enfin, dernier point de comparaison, on trouve aussi au musée du Caire l'idée de réserves ouvertes aux visiteurs sur demande, qui par leur organisation facilitent l'étude et la consultation des œuvres. Cela montre donc que Bosworth partage une même conception moderne des musées avec les principaux acteurs impliqués dans la mise en œuvre du plan Verne, ce qui a aussi, sans aucun doute, pu faciliter d'éventuels échanges entre eux.

Fig. 4

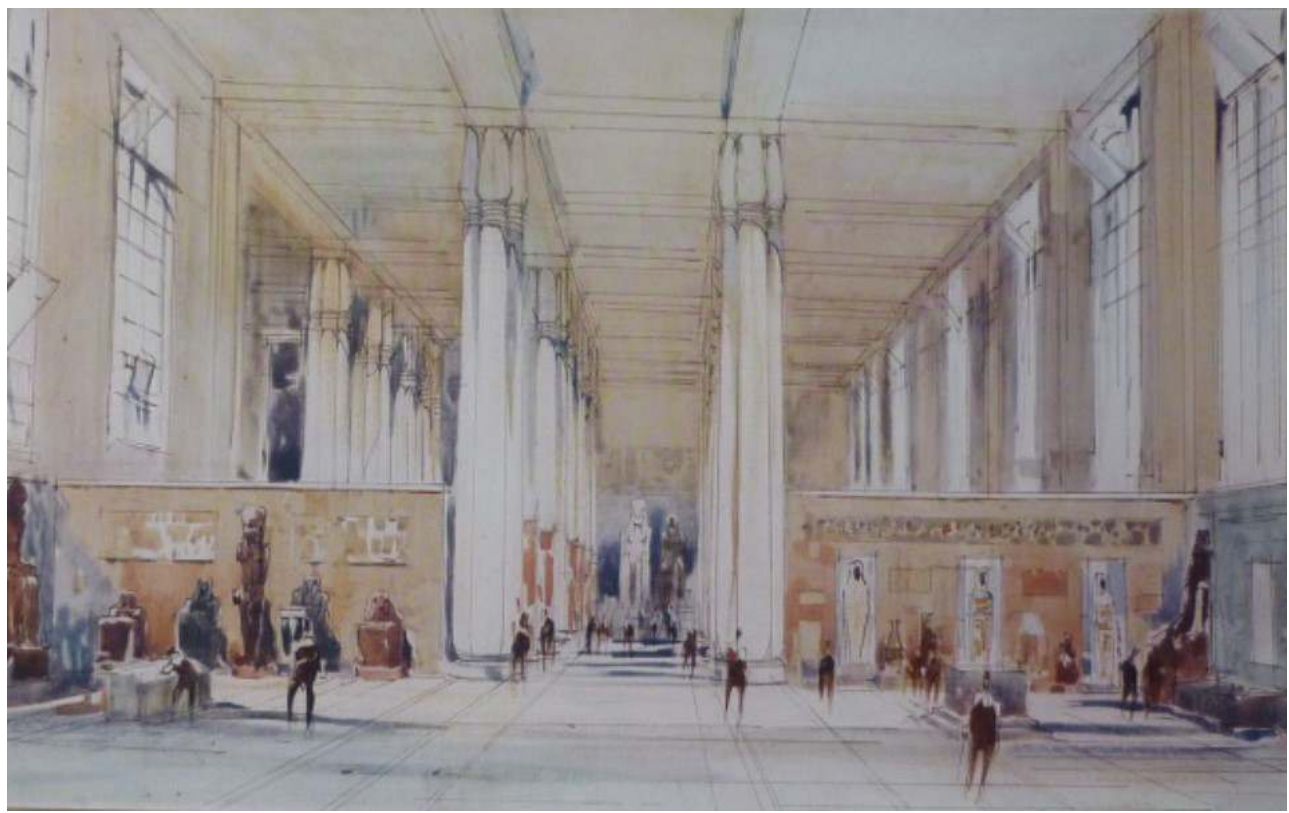

Intérieur suggéré d'une salle du musée du Caire, aquarelle de William Walcot d'après un dessin de Bosworth, 1925, dans The New Egyptian Museum and Research Institute at Cairo, planche 10, RAC, Folder 258A, Box 25, Series E, OMR - Office of the Messrs. Rockefeller 
Fig. 5

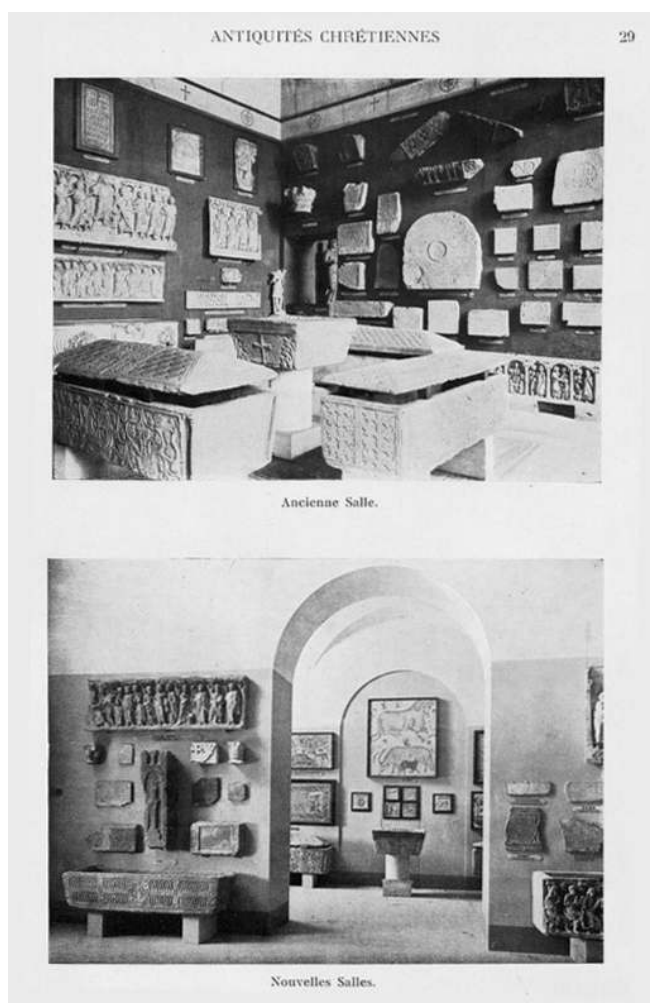

Ancienne et nouvelle salle consacrée aux antiquités chrétiennes, dans Bulletin des musées de France, 6e année, nº 1, janvier 1934, p. 29.

Il est donc très probable que du fait de sa proximité avec les principaux acteurs du projet et de sa bonne connaissance des musées américains de construction récente, Bosworth ait été sollicité pour donner son avis sur certains éléments architecturaux ou muséographiques du plan Verne. Toutefois, il n'était pas le seul contact étranger du directeur des Musées nationaux et en l'absence de traces écrites, il est difficile de définir avec plus de précisions le rôle exact de Bosworth dans ce projet, mais aussi de déterminer quels ont été les modèles étrangers utilisés pour réorganiser le Louvre. Cette contribution a toutefois pu apporter des éléments nouveaux concernant la recherche de financement pour le plan Verne et ouvrir une piste sur un probable apport américain dans la réorganisation du Louvre pendant l'entre-deux-guerres.

\section{NOTES}

1. Le ministère des Finances occupe toute l'aile côté rue de Rivoli (sur les trois étages) ainsi que le pavillon et l'aile de Flore; le musée des Arts décoratifs est installé dans le pavillon et l'aile Marsan ; le musée de la Marine occupe le second étage de la Cour carrée.

2. Paul Lorquet, «Que le musée du Louvre serait beau si l'on faisait mieux sa toilette. Et ne pourrait-on en faciliter la visite?», Quotidien, $1^{\mathrm{er}}$ juin 1929. 
3. Idem, ibidem.

4. Rapport mentionné dans Archives nationales (AN), 20144794/34, rapport d'Henri Verne au sous-secrétaire d'État aux Beaux-Arts, 7 novembre 1929, p. 5.

5. Idem, ibidem, p. 5.

6. Henri Verne, «L'avenir du musée du Louvre», L'Illustration, $\mathrm{n}^{\circ} 4529,21$ décembre 1929, pp. 766-768.

7. Pour plus de détails sur le plan Verne, voir Geneviève Bresc-Bautier, Guillaume Fonkenell, Françoise Mardrus, Histoire du Louvre, de la restauration à nos jours, Paris, Louvre éditions, Fayard, 2016, vol. 2, pp. 386-420.

8. Idem, ibidem, p. 768.

9. Médiathèque de l'architecture et du patrimoine (MAP), 80/3/20, rapport de Paul Léon au directeur de la comptabilité et du personnel du ministère des Finances, 22 décembre 1922.

10. AN, 20144794/34, lettre d'Henri Verne à Camille Lefèvre, s. d.

11. AN, 20144794/34, lettre d'Henri Verne à Paul Léon, 29 décembre 1928.

12. Massachusetts Institute of Technology (MIT), Institute Archives and Special Collections, Office of the President, Records of Julius A. Stratton, AC134, lettre de William Welles Bosworth (WWB) à Julius A. Stratton, 30 juin 1964 et 7 juillet 1964.

13. Rockefeller Archive Center (RAC), Folder 1251, Box 161, Series H, Office of the Messrs. Rockefeller (OMR), lettre d'Abraham Flexner à John D. Rockefeller Jr. (JDR Jr.), 27 mars 1929.

14. Idem, ibidem, mémorandum d'Arthur Woods à JDR Jr., $1^{\mathrm{er}}$ avril 1929.

15. Id., ibid., lettre de JDR Jr. à WWB, 28 mai 1929.

16. AN, 20144794/34, lettre de WWB à Henri Verne, 8 octobre 1929.

17. MIT, op. cit. note 12 .

18. Le carton d'invitation ne figure pas dans les archives mais la lettre de réponse de Bosworth se trouve dans AN, 20144781/2, lettre de WWB à Henri Verne, 5 février 1930.

19. RAC, Folder 354, Box 48, Series H, OMR, lettre de WWB à JDR Jr., 10 janvier 1930.

20. Correspondance et invitations relatives au University Club dans AN, AJ/16/6973.

21. Voir à ce sujet Jeffrey Abt, "The Breasted-Rockefeller Egyptian Museum Project: Philanthropy, Cultural Imperialism and National Resistance ", Art History, vol. 19, n 4 , décembre 1996, pp. 551-572.

22. RAC, Folder 258, Box 25, Series E, OMR, lettre de James Henry Breasted à JDR Jr., 24 août 1925.

23. RAC, Folder 258A, Box 25, Series E, OMR, The Egyptian Museum and Research Institute at Cairo, 1925.

24. Idem, ibidem, p. 23.

25. Id., ibid., p. 28.

\section{RÉSUMÉS}

Dès son arrivée à la tête des Musées nationaux en 1925, Henri Verne commence à mettre sur pied un vaste projet de réaménagement et de modernisation du Louvre, connu sous le nom de «plan Verne ». À la recherche d'un financement de 50 millions de francs pour permettre sa réalisation, il contacte William Welles Bosworth, architecte représentant John D. Rockefeller Jr. en France, pour qu'il l'aide à trouver un mécène américain. Si Rockefeller Jr. envisage un temps de prendre en charge le projet, il décide finalement de laisser cette responsabilité à l'État. Toutefois, il est 
probable que Bosworth, du fait de son expérience d'architecte et de sa bonne connaissance des musées américains, soit resté associé au plan Verne de manière informelle, en tant que conseiller. Les différentes pistes venant soutenir cette hypothèse seront présentées dans le but d'apporter un éclairage nouveau sur un éventuel apport américain dans la réorganisation du Louvre pendant l'entre-deux-guerres.

As soon as he became head of the Musées nationaux in 1925, Henri Verne began to put in place a vast project to reorganise and modernise the Louvre, known as the "plan Verne". In search of 50 million francs of financing, he contacted William Welles Bosworth, the architect who represented John D. Rockefeller Jr. in France, so that he could help him find an American sponsor. While Rockefeller Jr. envisaged taking the project on for a time, in the end he decided to leave the responsibility to the French government. However, it is likely that Bosworth, owing to his experience as an architect and his knowledge of American museums, remained informally associated with the "plan Verne" as an adviser. The various leads supporting this hypothesis will be presented with a view to throwing new light on an eventual American contribution to reorganisation of the Louvre during the interwar period.

\section{INDEX}

Mots-clés : Henri Verne, Paul Léon, William Welles Bosworth, John D. Rockefeller Jr., musée du Louvre, muséographie, financement, philanthropie, États-Unis

Keywords : Henri Verne, Paul Léon, William Welles Bosworth, John D. Rockefeller Jr., musée du Louvre, museography, financing, philanthropy, United States

\section{AUTEUR}

\section{ÉGLANTINE PASQUIER}

Églantine Pasquier est doctorante en histoire de l'architecture à l'université Paris 1 PanthéonSorbonne en co-encadrement avec l'École du Louvre. Ses recherches ont porté sur la sauvegarde du domaine de Versailles dans les années 1950 puis sur le mécénat américain à Versailles au Xx siècle. Sa thèse s'intitule Échanges culturels et architecturaux entre la France et les États-Unis : le rôle de l'architecte William Welles Bosworth (1869-1966), sous la direction de Jean-Philippe Garric et d'Alice Thomine-Berrada. Elle a bénéficié d'une bourse de recherche de l'École du Louvre pour l'année 2016-2017 ainsi que d'une grant-in-aid du Rockefeller Archive Center.

$* * *$

Églantine Pasquier is a PhD student in the history of architecture at the Université Paris 1 Panthéon-Sorbonne and the École du Louvre. Her research has focussed on the conservation of the grounds of Versailles in the 1950s, then on American sponsorship at Versailles in the twentieth century. Her thesis is titled Échanges culturels et architecturaux entre la France et les ÉtatsUnis : le rôle de l'architecte William Welles Bosworth (1869-1966), and is supervised by Jean-Philippe Garric and Alice Thomine-Berrada. She benefitted from a research grant from the École du Louvre for the year 2016-17 as well as a grant-in-aid from the Rockefeller Archive Center. 